\title{
Properties of the solution set of a generalized differential equation
}

\section{J.L. Davy}

We prove that the solution set of a generalized differential equation is connected and that points on the boundary of the solution funnel are peripherally attainable. This is done without the additional assumption of continuity in the state variable required in previous results. The result on upper semicontinuity of the solution set with respect to initial conditions is extended to include variations of initial time.

\section{Introduction}

In this paper we study the generalized differential equation

$$
\begin{aligned}
& x^{\prime}(t) \in F(t, x(t)) \text { almost everywhere } t \in I, \\
& x\left(t_{0}\right)=x_{0} .
\end{aligned}
$$

$E(t, x)$ is a convex compact set valued function which is upper semicontinuous in $x$ and bounded by an integrable function on the compact interval $I$. We assume that for a given $x$ there exists a measurable selector $f_{x}(t)$ contained in $F(t, x)$. The solution set of the equation, denoted by $H\left(t_{0}, x_{0}\right)$, is a nonempty compact subset of $C(I), \quad([11]$, $[10],[3],[7])$.

$H\left(t_{0}, x_{0}\right)$ is upper semicontinuous in $x_{0},([3],[8])$. We prove that $H\left(t_{0}, x_{0}\right)$ is upper semicontinuous in $\left(t_{0}, x_{0}\right)$. If $F$ is also

Received 10 December 1971. Communicated by W.A. Coppel. The author wishes to thank Mr W.A. Coppel, who suggested the problems considered in this paper. 
continuous in $x_{0}, H\left(t_{0}, x_{0}\right)$ is a connected subset of $C(I)$, every point on the boundary of the solution funnel is peripherally attainable and the generalized differential equation has the bang-bang property ([8], [9], [12]). We show that connectedness and peripheral attainability follow without the additional assumption of continuity. We give an example to show that the bang-bang property does not hold without continuity.

Filippov's paper [6] contains results similar to ours, but assumes the existence of a measurable selector $f(t, x)$ contained in $F(t, x)$ and does not contain proofs.

To make the paper self-contained we include results on upper semicontinuity due to Berge [2] and a proof of the existence of solutions.

\section{Upper semicontinuity}

Upper semicontinuity of compact set valued functions is a generalization of the concept of continuity of point valued functions. A point valued function is upper semicontinuous (as a set valued function) if and only if it is continuous. This section is based on Berge [2].

$X$ and $Y$ are topological spaces and $\Omega(Y)$ is the set of nonempty compact subsets of $Y$. $F$ maps from $X$ to $\Omega(Y)$. Let $A$ be a subset of $X$. Then

$$
F(A)=\bigcup_{x \in A} F(x)
$$

$F$ is upper semicontinuous at $x_{0}$ if for all open sets $G$ containing $F\left(x_{0}\right)$, there exists a neighbourhood $U\left(x_{0}\right)$ of $x_{0}$ such that $F\left(U\left(x_{0}\right)\right) \subseteq G . \quad F$ is upper semicontinuous if $F$ is upper semicontinuous at every point of $X$.

THEOREM 2.1. $F$ is upper semicontinuous if and only if $F^{+}(G)=\{x \mid F(x) \subseteq G\}$ is an open set for all open sets $G$ in $Y$.

Proof. (a) Assume $F$ is upper semicontinuous. Let $x_{0} \in F^{+}(G)$. There exists a neighbourhood $U\left(x_{0}\right)$ of $x_{0}$ such that $F\left(U\left(x_{0}\right)\right) \subseteq G$. Thus $U\left(x_{0}\right) \subseteq F^{+}(G)$ and $F^{+}(G)$ is an open set.

(b) Assume $G$ is open implies $F^{+}(G)$ is open. Let $x_{0} \in X$ and let 
$G$ be an open set containing $F\left(x_{0}\right) \cdot F^{+}(G)$ is a neighbourhood of $x_{0}$ and $F\left(F^{+}(G)\right) \subseteq G$. //

THEOREM 2.2. If $E$ is upper semicontinuous the image $F(K)$ of a compact set $K$ is compact.

Proof. Let $\left\{G_{i} \mid i \in I\right\}$ be an open covering of $E(K)$. If $x \in K$, the set $F(x)$, which is compact, is coverea by a finite number of $G_{i}$. Let their union be denoted by $G_{x} \cdot\left\{F^{+}\left(G_{x}\right) \mid x \in K\right\}$ is an open covering of $K$. Thus, there exists a finite subcovering $F^{+}\left(G_{x_{1}}\right), \ldots, F^{+}\left(G_{x_{n}}\right)$. $G_{x_{1}}, \ldots, G_{x_{n}}$ cover $F(K)$ and each $G_{x_{j}}$ is the union of a finite number of $G_{i}$. Therefore $F(K)$ is covered by a finite number of $G_{i}$ and thus is compact. //

THEOREM 2.3. If $F$ is upper semicontinuous, $K$ is a connected subset of $X$ and $F(x)$ is a connected subset of $Y$ for each $x \in K$ then $F(K)$ is a connected subset of $Y$.

Proof. Suppose $F(K)$ is not connected. That is, there exist two open disjoint sets $A_{1}, A_{2}$ of $Y$ such that $F(K) \subseteq A_{1} \cup A_{2}$ and $F(K) \cap A_{1} \neq \varnothing$ and $F(K) \cap A_{2} \neq \emptyset$. Then $F^{+}\left(A_{1}\right)$ and $F^{+}\left(A_{2}\right)$ are open sets of $X$. Let $x \in K$; then $F(x) \subseteq A_{1} \cup A_{2}$, and since $F(x)$ is connected, it is contained in $A_{1}$ or in $A_{2}$. Thus $K \subseteq F^{+}\left(A_{1}\right) \cup F^{+}\left(A_{2}\right)$. It is obvious that $F^{+}\left(A_{1}\right) \cap F^{+}\left(A_{2}\right)=\emptyset$, and $K \cap F^{+}\left(A_{1}\right) \neq \emptyset$ and $K \cap F^{+}\left(A_{2}\right) \neq \emptyset$. Thus $K$ is not connected, which is a contradiction. Therefore $\vec{F}(K)$ is connected. //

THEOREM 2.4. Let $F_{1}: X \rightarrow \Omega(Y)$ and $F_{2}: Y \rightarrow \Omega(Z)$ be upper semicontinuous. Define $F_{2} \circ F_{1}(x)=F_{2}\left(F_{1}(x)\right)$. Then $F_{2} \circ F_{1}$ maps $X$ to $\Omega(Z)$ and is upper semicontimuous.

Proof. By Theorem 2.2, $F_{2}\left(F_{1}(x)\right) \in \Omega(2)$. Let $G$ be an open set of 2 


$$
\begin{aligned}
\left(F_{2} \circ F_{1}\right)^{+}(G) & =\left\{x \mid F_{2} \circ E_{1}(x) \subseteq G\right\} \\
& =\left\{x \mid F_{1}(x) \subseteq F_{2}^{+}(G)\right\} \\
& =F_{1}^{+}\left[F_{2}^{+}(G)\right],
\end{aligned}
$$

which is open in $X$ by Theorem 2.1. Thus $F_{2} \circ F_{1}$ is upper semicontinuous by Theorem 2.1. //

Let $X$ be a metric space, $\varepsilon$ a positive real number and $x \in X$. Define $B_{\varepsilon}(x)=\{y \mid d(x, y)<\varepsilon\}$. Let $A$ be a subset of $X$. Define $A^{\varepsilon}=\underset{x \in A}{\bigcup} B_{\varepsilon}(x)$

THEOREM 2.5. Let $X$ and $Y$ be metric spaces and $F: X \rightarrow \Omega(Y)$. $F$ is upper semicontinuous at $x_{0}$ if and only if for each $\varepsilon>0$ there exists $\delta>0$ such that $F\left(B_{\delta}\left(x_{0}\right)\right) \subseteq F^{\varepsilon}\left(x_{0}\right)$.

Proof. (a) Suppose $F$ is upper semicontinuous at $x_{0}$. Choose a neighbourhood $U\left(x_{0}\right)$ of $x_{0}$ such that $E\left(U\left(x_{0}\right)\right) \subseteq F^{\varepsilon}\left(x_{0}\right)$. Choose $\delta>0$ such that $B_{\delta}\left(x_{0}\right) \subseteq U\left(x_{0}\right)$. Therefore $F\left(B_{\delta}\left(x_{0}\right)\right) \subseteq E^{\varepsilon}\left(x_{0}\right)$.

(b) Let $G$ be an open set of $Y$ containing $F\left(x_{0}\right)$. Suppose there does not exist $\varepsilon>0$ such that $E^{\varepsilon}\left(x_{0}\right) \subseteq G$. Then there exists $x_{k} \in F^{l / k}\left(x_{0}\right)$ such that $x_{k} \notin G$. Now $x_{k} \in \overline{F^{l}\left(x_{0}\right)}$ which is a compact set. Therefore there exists a subsequence, also denoted by $\left\{x_{k}\right\}$, converging to a point $x_{\infty} \in \overline{F^{l}\left(x_{0}\right)}$. But $x_{k} \in F^{1 / p}\left(x_{0}\right)$ for all $k \geq p$. Thus $x_{\infty} \in \overline{F^{l / P}\left(x_{0}\right)}$ for all $p$. Therefore $x_{\infty}$ is contained in $F\left(x_{0}\right)$. But $x_{k} \in G^{c}$, which is closed. Hence $x_{\infty} \in G^{c}$. This contradiction implies that there exists $\varepsilon>0$ such that $F^{\varepsilon}\left(x_{0}\right) \subseteq G$. Choose $\delta>0$ such that $F\left(B_{\delta}\left(x_{0}\right)\right) \subseteq F^{\varepsilon}\left(x_{0}\right) \subseteq G$. Put $u\left(x_{0}\right)=B_{\delta}\left(x_{0}\right)$. / /

Let $X$ be a normed linear space. A subset $K$ of $X$ is said to be 
convex if, given $x_{1}$ and $x_{2} \in K$, all points of the form $\alpha x_{1}+(1-\alpha) x_{2}$ with $0 \leq \alpha \leq 1$ are in $K$. If $A$ is a subset of $X$, the convex hull of $A$, denoted by $\operatorname{co} A$, is the smallest convex set containing $A$. Since the intersection of any collection of convex sets is a convex set, $\operatorname{co} A$ is the intersection of all the convex sets containing $A$. The closed convex hull of $A$, denoted by $\overline{\operatorname{co} A}$, is defined by $\overline{\operatorname{co} A}=\overline{(\operatorname{co} A)}$.

LEMMA 2.6. $\operatorname{co}\left(A^{\varepsilon}\right)=(\operatorname{coA})^{\varepsilon}$.

Proof. (a) Let $x \in \operatorname{co}\left(A^{\varepsilon}\right)$. Then $x=\lambda x_{1}+(1-\lambda) x_{2}$ where $x_{1}, x_{2} \in A^{\varepsilon}$ and $0 \leq \lambda \leq I$. Thus there exist $y_{1}$ and $y_{2} \in A$ such that $\left\|x_{1}-y_{1}\right\|<\varepsilon$ and $\left\|x_{2}-y_{2}\right\|<\varepsilon$. Put $y=\lambda y_{1}+(1-\lambda) y_{2}$. Then $y \in \operatorname{co} A$ and

$$
\|x-y\|=\left\|\lambda\left(x_{1}-y_{1}\right)+(1-\lambda)\left(x_{2}-y_{2}\right)\right\|<\lambda \varepsilon+(1-\lambda) \varepsilon=\varepsilon .
$$

Therefore $x \in(C O A)^{\varepsilon}$.

(b) Let $x \in(C O A)^{\varepsilon}$. Then there exists $y \in \operatorname{coA}$ such that $\|x-y\|_{i}<\varepsilon$. Therefore $y=\lambda y_{1}+(1-\lambda) y_{2}$ where $y_{1}$ and $y_{2} \in A$ and $0 \leq \lambda \leq 1$. Put $z=x-y, x_{1}=y_{1}+z$ and $x_{2}=y_{2}+z$. Then $x_{1}$ and $x_{2} \in A^{\varepsilon}$ and $\lambda x_{1}+(1-\lambda) x_{2}=x$. Thus $x \in \operatorname{co}\left(A^{\varepsilon}\right)$. //

COROLLARY 2.7. $\overline{\operatorname{CO} A}=\operatorname{co}(\bar{A})$.

THEOREM 2.8. Let $X$ be a metric space and $Y$ a normed linear space. Suppose $F: X \rightarrow \Omega(Y)$ is upper semicontinuaus at $x_{0}$. If $x_{k}$ tends to $x_{0}$ then

$$
\prod_{i=1}^{\infty} \overline{\operatorname{co}} \bigcup_{k=i}^{\infty} F\left(x_{k}\right) \subseteq \operatorname{coF}\left(x_{0}\right) .
$$

Proof. Let $\varepsilon>0$. Choose $\delta>0$ such that $F\left(B_{\delta}\left(x_{0}\right)\right) \subseteq F^{\varepsilon}\left(x_{0}\right)$. Choose $k_{0}$ such that $k \geq k_{0}$ implies $x_{k} \in B_{\delta}\left(x_{0}\right)$. Therefore $k \geq k_{0}$ 
implies $F\left(x_{k}\right) \subseteq F^{\varepsilon}\left(x_{0}\right)$ and $\bigcup_{k=k_{0}}^{\infty} F\left(x_{k}\right) \subseteq F^{\varepsilon}\left(x_{0}\right)$. Thus

$$
\text { co } \bigcup_{k=k_{0}}^{\infty} F\left(x_{k}\right) \subseteq \operatorname{coF}^{\varepsilon}\left(x_{0}\right)=\left(\operatorname{coF}\left(x_{0}\right)\right)^{\varepsilon} \text {. }
$$

Therefore

$$
\overline{c o} \bigcup_{k=k_{0}}^{\infty} F\left(x_{k}\right) \subseteq \overline{\left(\operatorname{co} F\left(x_{0}\right)\right)^{\varepsilon}} \text {. }
$$

Hence

$$
\bigcap_{i=1}^{\infty} \overline{\operatorname{co}} \bigcup_{k=i}^{\infty} F\left(x_{k}\right) \subseteq \overline{\left(\cos \left(x_{0}\right)\right)^{\varepsilon}} \text {. }
$$

Thus

$$
\bigcap_{i=1}^{\infty} \overline{c o} \bigcup_{k=i}^{\infty} F\left(x_{k}\right) \subseteq \operatorname{coF}\left(x_{0}\right)
$$

since $\operatorname{coF}\left(x_{0}\right)$ is closed by Corollary 2.7. //

\section{Generalized differential equations}

We will consider the generalized differential equation

$$
\begin{aligned}
& x^{\prime}(t) \in F(t, x(t)) \text { almost everywhere } t \in I, \\
& x\left(t_{0}\right)=x_{0},
\end{aligned}
$$

where $F$ satisfies the following conditions:

1. $F$ maps from $I \times R^{n}$ into $\Omega\left(R^{n}\right)$ where $I$ is the compact interval $[a, b]$;

2. $F(t, x)$ is convex;

3. for all $t \in I, x \mapsto F(t, x)$ is upper semicontinuous on $R^{n}$;

4. for all $x \in R^{n}$ there exists $f_{x}: I \rightarrow R^{n}$ such that $f_{x}$ is measurable and $f_{x}(t) \in F(t, x)$; 
5. there exists $g \in L^{1}(I)$ such that $y \in F(t, x)$ implies $|y| \leq g(t)$.

Let $t_{0} \in I$ and $x_{0} \in R^{n}$. The function $x$ is a solution of the generalized differential equation if and only if

$$
\begin{aligned}
& \text { (i) } x: I \rightarrow R^{n} \text { is absolutely continuous, } \\
& \text { (ii) } x^{\prime}(t) \in F(t, x(t)) \text { almost everywhere } t \in I, \\
& \text { (iii) } x\left(t_{0}\right)=x_{0} .
\end{aligned}
$$

The set of all solutions is called the solution set and is denoted by $H\left(t_{0}, x_{0}\right)$. In this paper we will study the properties of the solution set.

\section{Existence}

In this section we give Aumann's proof [1] of a result on the convergence of absolutely continuous functions and use this result in Kikuchi's proof [7] of the existence of a solution.

THEOREM 4.1. Let $\left\{x_{k}\right\}$ be a sequence of absolutely continuous functions $x_{k}: I \rightarrow R^{n}$. We suppose that

$$
\begin{aligned}
& \text { (i) } x_{k}(t) \rightarrow x(t) \text { as } k+\infty \text { for alz } t \in I \text { where } x: I \rightarrow R^{n} \text {, } \\
& \text { (ii) }\left|x_{k}^{\prime}(t)\right| \leq g(t) \text { almost everywhere } t \in I \text { where } g: I \rightarrow R \text { is } \\
& \text { an integrable function. }
\end{aligned}
$$

Then $x$ is an absolutely continuous function such that

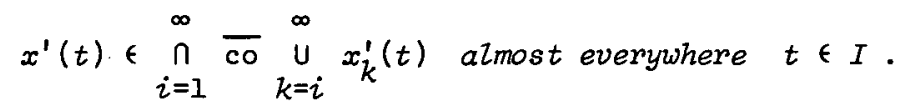

Proof. Since $x_{k}$ is absolutely continuous, $x_{k}^{\prime} \in L^{l}(I)$. Also $\left|x_{k}^{\prime}(t)\right| \leq g(t)$ almost everywhere $t \in I$. Therefore by Theorem IV.8.9 on page 292 of Dunford and Schwartz [5] there exists a subsequence $\left\{x_{k(j)}^{\prime}\right\}$ 
converging weakly to $f \in L^{l}(I)$. Thus

$$
\begin{aligned}
x(t) & =\lim _{j \rightarrow \infty} x_{k(j)}(t) \\
& =\lim _{j \rightarrow \infty}\left[x_{k(j)}(a)+\int_{a}^{t} x_{k(j)}^{\prime}\right] \\
& =x(a)+\int_{a}^{t} f .
\end{aligned}
$$

Therefore $x$ is absolutely continuous and $x^{\prime}(t)=f(t)$ almost everywhere $t \in I$.

By Corollary V.3.14 on page 422 of Dunford and Schwartz [5], there exists a sequence $\left\{g_{m}\right\}$ of convex combinations of $\left\{x_{k(1)}^{\prime}, x_{k(2)}^{\prime}, \ldots\right\}$ converging strongly to $f$. There exists a subsequence, also denoted by $\left\{g_{m}\right\}$, such that $g_{m}(t) \rightarrow f(t)$ almost everywhere $t \in I$. But

$$
\begin{aligned}
g_{m}(t) & \in \operatorname{co} \bigcup_{j=1}^{\infty} x_{k(j)}^{\prime}(t) \\
& \subseteq \operatorname{co} \bigcup_{k=1}^{\infty} x_{k}^{\prime}(t) .
\end{aligned}
$$

Hence

$$
f(t) \in \overline{c o} \bigcup_{k=1}^{\infty} x_{k}^{\prime}(t) \text { almost everywhere } t \in I \text {. }
$$

But $\left\{x_{i}, x_{i+1}, \ldots\right\}$ also tends to $x$ for all positive integers $i$. Thus

$$
f(t) \in \overline{c o} \bigcup_{k=i}^{\infty} x_{k}^{\prime}(t) \text { almost everywhere } t \in I
$$

Therefore

$$
f(t) \epsilon \prod_{i=1}^{\infty} \overline{\text { co }} \bigcup_{k=i}^{\infty} x_{k}^{\prime}(t) \text { almost everywhere } t \in I . \quad / /
$$

THEOREM 4.2. $H\left(t_{0}, x_{0}\right)$ is nonempty. 
Proof. Subdivide $\left[t_{0}, b\right]$ into $k$ equal parts by $t_{i}=t_{0}+i \frac{b-t_{0}}{k}$. We define $x_{k}:\left[t_{0}, b\right] \rightarrow R^{n}$ inductively. First $x_{k}\left(t_{0}\right)=x_{0}$. Suppose $x_{k}$ is defined up to $t_{i}$ where $0 \leq i<k$. Select a measurable function $f_{i}:\left[t_{i}, t_{i+1}\right] \rightarrow R^{n}$ such that $f_{i}(t) \in F\left(t, x_{k}\left(t_{i}\right)\right)$ for all $t \in\left[t_{i}, t_{i+1}\right]$. Define

$$
x_{k}(t)=x_{k}\left(t_{i}\right)+\int_{t_{i}}^{t} f_{i} \text { for all } t \in\left[t_{i}, t_{i+1}\right] .
$$

Define $f:\left[t_{0}, b\right] \rightarrow R^{n}$ by $f(t)=f_{i}(t)$ for all $t \in\left[t_{i}, t_{i+1}\right]$. Hence

$$
\begin{gathered}
x_{k}(t)=x_{0}+\int_{t_{0}}^{t} f, \\
\left|x_{k}(t)-x_{0}\right| \leq \int_{t_{0}}^{t} g, \\
\left|x_{k}(t)\right| \leq\left|x_{0}\right|+\int_{t_{0}}^{b} g .
\end{gathered}
$$

Therefore $x_{k}$ is well defined.

Now

$$
x_{k}(t)=f(t) \text { almost everywhere } t \in\left[t_{0}, b\right] .
$$

Thus

$$
\left|x_{k}(t)\right| \leq g(t) \text { almost everywhere } t \in\left[t_{0}, b\right] .
$$

Therefore $\left\{x_{k}\right\}$ is equicontinuous. Define $y_{k}:\left[t_{0}, b\right]+R^{n}$ by $y_{k}(t)=x_{k}\left(t_{i}\right)$ if $t \in\left[t_{i}, t_{i+1}\right]$. Then $x_{k}^{\prime}(t) \in F\left(t, y_{k}(t)\right)$ almost everywhere $t \in\left[t_{0}, b\right]$.

Consider the sequence $\left\{x_{k}\right\}$. It is bounded and equicontinuous. Thus 
by Ascoli's Theorem it has a convergent subsequence, also denoted by $\left\{x_{k}\right\}$, converging to $x \in C(I)$. By Theorem 4.1, $x$ is absolutely continuous and

$$
\begin{aligned}
x^{\prime}(t) & \in \bigcap_{i=1}^{\infty} \overline{c o} \underset{k=i}{U} x_{k}^{\prime}(t) \text { almost everywhere } t \in\left[t_{0}, b\right] \\
& \subseteq \bigcap_{i=1}^{\infty} \overline{c o} \underset{k=i}{U} F\left(t, y_{k}(t)\right) \text { almost everywhere } t \in\left[t_{0}, b\right] \\
& \subseteq F(t, x(t)) \text { almost everywhere } t \in\left[t_{0}, b\right]
\end{aligned}
$$

by Theorem 2.8 since $y_{k}(t)$ tends to $x(t)$. Also

$$
x\left(t_{0}\right)=\lim _{k \rightarrow \infty} x_{k}\left(t_{0}\right)=x_{0} .
$$

Similarly-we can find $x:\left[a, t_{0}\right] \rightarrow R^{n}$ such that $x\left(t_{0}\right)=x_{0}$ and $x^{\prime}(t) \in F(t, x(t))$ almost everywhere $t \in\left[a, t_{0}\right]$. Putting these two functions together we have $x:[a, b] \rightarrow R^{n}$ and $x \in H\left(t_{0}, x_{0}\right)$. Thus $H\left(t_{0}, x_{0}\right)$ is nonempty. //

\section{Compactness and upper semicontinuity}

In this section we prove that the solution set is compact and upper semicontinuous.

THEOREM 5.1. If $M \in \Omega\left(I \times R^{n}\right)$, then $H(M)$ is a compact subset of $C(I)$.

Proof. (a) Let $x \in H(M)$. There exists $t_{0} \in I$ such that $\left(t_{0}, x\left(t_{0}\right)\right) \in M$. Let $M^{\prime}$ be the projection of $M$ into $R^{n}$. $M^{\prime}$ is compact and $x\left(t_{0}\right) \in M^{\prime}$. Thus $\left|x\left(t_{0}\right)\right|<d$, where $d$ is a bound for $M^{\prime}$. Now $x(t)=x\left(t_{0}\right)+\int_{t_{0}}^{t} x^{\prime}$. Therefore $\left|x(t)-x\left(t_{0}\right)\right| \leq \int_{I} g$. Hence $\|x\| \leq d+\int_{I} g$. Thus $H(M)$ is bounded.

(b) If $x \in H(M)$ we have $\left|x^{\prime}(t)\right| \leq g(t)$ almost everywhere $t \in I$. 
Thus $\left|x(t)-x\left(t_{0}\right)\right| \leq \int_{t_{0}}^{t} g$. Therefore $H(M)$ is equicontinuous.

(c) Let $x_{k} \rightarrow x$ where $x_{k} \in H(M)$. By Theorem 4.1, $x$ is absolutely continuous and

$$
\begin{aligned}
& x^{\prime}(t) \in \prod_{i=1}^{\infty} \overline{c o} \bigcup_{k=i}^{\infty} x_{k}^{\prime}(t) \text { almost everywhere } t \in I \\
& \subseteq \bigcap_{i=1}^{\infty} \overline{c o} \bigcup_{k=i}^{\infty} F\left(t, x_{k}(t)\right) \text { almost everywhere } t \in I \\
& \subseteq F(t, x(t)) \text { almost everywhere } t \in I
\end{aligned}
$$

by Theorem 2.8 .

For each $x_{k}$ there exists $t_{k} \in I$ such that $\left(t_{k}, x_{k}\left(t_{k}\right)\right) \in M$. Since $M$ is compact there exists a subsequence, also denoted by $\left\{\left(t_{k}, x_{k}\left(t_{k}\right)\right)\right\}$, such that $\left(t_{k}, x_{k}\left(t_{k}\right)\right)$ tends to $\left(t_{0}, x_{0}\right) \in M$. Let $\varepsilon>0$. Choose $k_{0}$ such that $k \geq k_{0}$ implies $\left\|x-x_{k}\right\|<\frac{\varepsilon}{3}$, and $\delta>0$ such that $\left|t-t_{0}\right|<\delta$ implies $\left|x(t)-x\left(t_{0}\right)\right|<\frac{\varepsilon}{3}$. Choose $k_{1} \geq k_{0}$ such that $k \geq k_{1}$ implies $\left|t_{k}-t_{0}\right|<\delta$ and $\left|x_{k}\left(t_{k}\right)-x_{0}\right|<\frac{\varepsilon}{3}$. Therefore $\left|x\left(t_{0}\right)-x_{0}\right| \leq\left|x\left(t_{0}\right)-x\left(t_{k}\right)\right|+\left|x\left(t_{k}\right)-x_{k}\left(t_{k}\right)\right|+\left|x_{k}\left(t_{k}\right)-x_{\theta}\right|$ $<\frac{\varepsilon}{3}+\frac{\varepsilon}{3}+\frac{\varepsilon}{3}=\varepsilon$.

/Since $\varepsilon$ is any positive number we have $x\left(t_{0}\right)=x_{0}$. Thus $x \in H(M)$ and $H(M)$ is closed in $C(I)$.

(d) Thus by Ascoli's Theorem, $H(M)$ is compact in $C(I)$. // COROLLARY 5.2. $\quad H$ maps from $I \times R^{n}$ into $\Omega(C(I))$. Proof. Theorems 4.2 and 5.1 give the result. // We now use Theorem 5.1 to prove that $B$ is upper. semicontinuous. THEOREM 5.3. $H: I \times R^{n} \rightarrow \Omega(C(I))$ is an upper semicontinuous map. 
Proof. Let $\left(t_{0}, x_{0}\right) \in I \times R^{n}$. Assume that $H$ is not upper semicontinuous at $\left(t_{0}, x_{0}\right)$; that is, there exists $\varepsilon_{0}>0$ such that for all $\delta>0, H\left(B_{\delta}\left(t_{0}, x_{0}\right)\right) \notin{ }_{H}^{\varepsilon_{0}}\left(t_{0}, x_{0}\right)$. Choose $x_{k}$ such that $x_{k} \in H\left(B_{1 / k}\left(t_{0}, x_{0}\right)\right)$ and $x_{k} \notin H^{\varepsilon_{0}}\left(t_{0}, x_{0}\right)$. Now $x_{k} \in H \sqrt{\left.B_{1}\left(t_{0}, x_{0}\right)\right]}$, which is compact by Theorem 5.1. There exists a subsequence, also denoted by $\left\{x_{k}\right\}$, such that $x_{k}$ converges to $x \in H\left(B_{1}\left(t_{0}, x_{0}\right)\right]$. But there exists $t_{k} \in I$ such that $\left(t_{k}, x_{k}\left(t_{k}\right)\right) \in B_{1 / k}\left(t_{0}, x_{0}\right)$. Let $\varepsilon>0$. Choose $k_{0}$ such that $k \geq k_{0}$ implies $\left\|x-x_{k}\right\|<\frac{\varepsilon}{3}$ and $\delta>0$ such that $\left|t-t_{0}\right|<\delta$ implies $\left|x(t)-x\left(t_{0}\right)\right|<\frac{\varepsilon}{3}$. Choose $k_{1} \geq k_{0}$ such that $k \geq k_{1}$ implies $\left|t_{k}-t_{0}\right|<\delta$ and $\left|x_{k}\left(t_{k}\right)-x_{0}\right|<\frac{\varepsilon}{3}$. Therefore $\left|x\left(t_{0}\right)-x_{0}\right| \leq\left|x\left(t_{0}\right)-x\left(t_{k}\right)\right|+\left|x\left(t_{k}\right)-x_{k}\left(t_{k}\right)\right|+\left|x_{k}\left(t_{k}\right)-x_{0}\right|<\frac{\varepsilon}{3}+\frac{\varepsilon}{3}+\frac{\varepsilon}{3}=\varepsilon$.

Since $\varepsilon$ is any positive number we have $x\left(t_{0}\right)=x_{0}$. Thus $x \in H\left(t_{0}, x_{0}\right)$.

But $x_{k} \notin H^{\varepsilon_{0}}\left(t_{0}, x_{0}\right)$. Therefore $x \notin H\left(t_{0}, x_{0}\right)$. From this contradiction we conclude that $H$ is upper semicontinuous. //

\section{Connectedness}

In this section we prove that the solution set is connected.

LEMMA 6.1. Let $\left(t_{0}, x_{0}\right) \in I \times R^{n}$. Suppose $F$ does not depend on $x$. Then $H\left(t_{0}, x_{0}\right)$ is convex.

Proof. Let $x_{1}$ and $x_{2} \in H\left(t_{0}, x_{0}\right)$; that is $x_{i}\left(t_{0}\right)=x_{0}$ and

$$
x_{i}^{\prime}(t) \in F(t) \text { almost everywhere } t \in I \text { for } i=1,2 .
$$

Consider $x=\lambda x_{1}+(1-\lambda) x_{2}, 0 \leq \lambda \leq 1$. We have 


$$
x\left(t_{0}\right)=\lambda x_{0}+(I-\lambda) x_{0}=x_{0}
$$

and

$$
\begin{aligned}
x^{\prime}(t) & =\lambda x_{1}^{\prime}(t)+(1-\lambda) x_{2}^{\prime}(t), \\
& \in F(t) \text { almost everywhere } t \in I,
\end{aligned}
$$

since $F$ is convex. Thus $H\left(t_{0}, x_{0}\right)$ is convex. //

LEMMA 6.2. Suppose $F$ depends on a parameter $u \in R^{m}$ and that $F$ is upper semicontinuous in $(x, u)$. Let $H\left(t_{0}, x_{0}, u_{0}\right)$ denote the solution set passing through $\left(t_{0}, x_{0}\right)$ when $u=u_{0}$. Then

$H: I \times R^{n+m} \rightarrow C(I)$ is upper semicontinuous.

Proof. We consider the system

$$
\begin{aligned}
& x^{\prime} \in F(t, x) \\
& u^{\prime}=0 ;
\end{aligned}
$$

that is, $\tilde{x}^{\prime} \in \tilde{F}(t, x)$ where $\tilde{x}=\left(\begin{array}{l}x \\ u\end{array}\right)$ and $\tilde{F}=\left(\begin{array}{l}F \\ 0\end{array}\right)$. The solution set of this system $\tilde{H}\left(t_{0}, x_{0}, u_{0}\right)$ is upper semicontinuous by Theorem 5.3. Define $p: C_{R^{n+m}}(I)+C_{R^{n}}(I)$ by $p(\tilde{x})(t)=q \circ \tilde{x}(t)$ where $q$ is the projection operator $q: R^{n+m} \rightarrow R^{n}$. Then $p$ is continuous, and $H\left(t_{0}, x_{0}, u_{0}\right)=p \circ \tilde{H}\left(t_{0}, x_{0}, u_{0}\right)$. Therefore $H$ is upper semicontinuous by Theorem 2.4. $/ /$

Subdivide $I=[a, b]$ into $k$ equal parts by $t_{i}=a+i \frac{b-a}{k}$. We define a function $A_{i}: C\left[t_{0}, t_{i}\right] \rightarrow \Omega\left(C\left[t_{0}, t_{i+1}\right]\right), i=0, \ldots, k-1$. Let $x \in C\left[t_{0}, t_{i}\right]$. Then $y \in A_{i}(x)$ if and only if

(i) $y:\left[t_{0}, t_{i+1}\right]+R^{n}$;

(ii) $y(t)=x(t)$ for all $t \in\left[t_{0}, t_{i}\right]$;

(iii) $y$ is absolutely continuous on $\left[t_{i}, t_{i+1}\right]$; 
(iv) $y^{\prime}(t) \in F\left(t, x\left(t_{i}\right)\right)$ almost everywhere $t \in\left[t_{i}, t_{i+1}\right]$.

LEMMA 6.3. $A_{i}(x)$ is a compact convex nonempty set. $A_{i}: C\left[t_{0}, t_{i}\right] \rightarrow \Omega\left(C\left[t_{0}, t_{i+1}\right]\right)$ is upper semicontinuous.

Proof. Consider the equation

$$
\begin{aligned}
& y^{\prime}(t) \in F\left(t, x\left(t_{i}\right)\right) \text { almost everywhere } t \in\left[t_{i}, t_{i+1}\right], \\
& y\left(t_{i}\right)=x\left(t_{i}\right) .
\end{aligned}
$$

The right-hand side does not depend on $y$. Thus by Lemma 6.1 and Theorems 4.2 and $5.1, A_{i}(x)$ is a compact convex nonempty set.

By Lemma 6.2, $H\left(t_{i}, x\left(t_{i}\right), x\left(t_{i}\right)\right)$ is upper semicontinuous. Let $\varepsilon>0$. Choose $\delta>0$ such that $\left|(t, u, v)-\left(t_{i}, x_{0}\left(t_{i}\right), x_{0}\left(t_{i}\right)\right)\right|<2 \delta$ implies $H(t, u, v) \subseteq H^{\varepsilon}\left(t_{i}, x_{0}\left(t_{i}\right), x_{0}\left(t_{i}\right)\right)$ and such that $\delta<\varepsilon$. Therefore $\left\|x-x_{0}\right\|<\delta$ implies $\left|x(t)-x_{0}(t)\right|<\varepsilon$ for all $t \in\left[t_{0}, t_{i}\right]$ and $H\left(t_{i}, x\left(t_{i}\right), x\left(t_{i}\right)\right) \subseteq H^{\varepsilon}\left(t_{i}, x_{0}\left(t_{i}\right), x_{0}\left(t_{i}\right)\right)$; that is, $\left\|x-x_{0}\right\|<\delta$ implies $A_{i}(x) \subseteq A_{i}^{\varepsilon}\left(x_{0}\right)$. //

Let $y \in C(I)$. Define a function

$$
b_{y i}:\left[t_{i}, t_{i+1}\right] \rightarrow \Omega\left(c\left[t_{0}, t_{i+1}\right]\right), i=0, \ldots, k-1 .
$$

Let $r \in\left[t_{i}, t_{i+1}\right]$. Then $z \in b_{y i}(r)$ if and only if

$$
\begin{aligned}
& \text { (i) z: }\left[t_{0}, t_{i+1}\right] \rightarrow R^{n} ; \\
& \text { (ii) } z(t)=y(t) \text { if } t \in\left[t_{0}, r\right] ; \\
& \text { (iii) } z \text { is absolutely continuous on }\left[r, t_{i+1}\right] ; \\
& \text { (iv) } z^{\prime}(t) \in F\left(t, y\left(t_{i}\right)\right) \text { almost everywhere } t \in\left[r, t_{i+1}\right] \text {. }
\end{aligned}
$$

LEMMA 6.4. $b_{y i}(r)$ is a compact convex nonempty set. 
$b_{y i}:\left[t_{i}, t_{i+1}\right] \rightarrow \Omega\left(c\left[t_{0}, t_{i+1}\right]\right)$ is upper semicontinuous.

Proof. Consider the equation

$$
\begin{aligned}
& z^{\prime}(t) \in F\left(t, y\left(t_{i}\right)\right) \text { almost everywhere } t \in\left[t_{i}, t_{i+1}\right], \\
& z(r)=y(r) .
\end{aligned}
$$

The right hand side does not depend on $z$. Thus by Lemma 6.1 and Theorems 4.2 and 5.1, $b_{y i}(r)$ is a compact convex nonempty set. By Theorem 5.3, $H(r, a)$ is upper semicontinuous. Let $\varepsilon>0$. Choose $\gamma>0$ such that $\left|(r, a)-\left(r_{0}, a_{0}\right)\right|<2 \gamma$ implies $H(r, a) \subseteq H^{\varepsilon}\left(r_{0}, a_{0}\right)$. Choose $\delta_{1}>0$ such that $\left|r-r_{0}\right|<\delta_{1}$ implies $\left|y(r)-y\left(r_{0}\right)\right|<\gamma$ and such that $\delta_{1}<\gamma$. Thus $\left|r-r_{0}\right|<\delta_{1}$ implies $H(r, y(r)) \subseteq H^{\varepsilon}\left(r_{0}, y\left(r_{0}\right)\right)$. The set of all solutions is an equicontinuous family. Choose $\delta_{2}>0$ such that $\left|r-r_{0}\right|<\delta_{2}$ implies $\left|z(r)-z\left(r_{0}\right)\right|<\frac{\varepsilon}{2}$ for all solutions $z$. Choose $\delta_{3}>0$ such that $\left|r-r_{0}\right|<\delta_{3}$ implies $\left|y(r)-y\left(r_{0}\right)\right|<\frac{\varepsilon}{2}$. Put $\delta=\min \left\{\delta_{1}, \delta_{2}, \delta_{3}\right\}$. Then $\left|r-r_{0}\right|<\delta$ implies $b_{y i}(r) \subseteq b_{y i}^{\varepsilon}(r)$. $/ 1$ Define a function $B_{y i}:\left[t_{i}, t_{i+1}\right] \rightarrow \Omega(C(I)), i=0, \ldots, k-1$, by $B_{y i}(t)=A_{k-1} \circ \ldots \circ A_{i+1} \circ b_{y i}(t)$. Define a function $B_{y}: I \rightarrow \Omega(C(I))$ by $B_{y}(t)=B_{y i}(t)$ for all $t \in\left[t_{i}, t_{i+1}\right]$.

LEMMA 6.5. $B_{y}(t)$ is a compact connected nonempty set. $B_{y}: I \rightarrow \Omega(C(I))$ is upper semicontinuous.

Proof. First we note that a convex set is connected. By Lemmas 6.3 and 6.4 and Theorems $2.2,2.3$ and 2.4 we have that $B_{y i}(t)$ is a compact connected nonempty set and that $B_{y i}$ is upper semicontinuous. Now $B_{y i}\left(t_{i+1}\right)=B_{y(i+1)}\left(t_{i+1}\right), i=0, \ldots, k-2$. Hence $B_{y}$ is well defined and upper semicontinuous.

Having defined the operator $B_{y}$ we can now mimic the standard proof 
of Kneser's Theorem, (see Coppel [4]).

THEOREM 6.6. Let $\left(t_{0}, x_{0}\right) \in I \times R^{n}$. Then $H\left(t_{0}, x_{0}\right)$ is a connected subset of $C(I)$.

Proof. If $\left.H\left(t_{0}, x_{0}\right)\right|_{\left[t_{0}, b\right]}$ and $\left.H\left(t_{0}, x_{0}\right)\right|_{\left[a, t_{0}\right]}$ are connected then $H\left(r_{0}, x_{0}\right)$ is connected. So without loss of generality we can assume that $t_{0}=a$.

Suppose that $H\left(a, x_{0}\right)$ is disconnected; that is, $H\left(a, x_{0}\right)=H_{1} \cup H_{2}$ where $H_{1}$ and $H_{2}$ are disjoint nonempty closed sets. Let $x_{1} \in H_{1}$ and $x_{2} \in H_{2}$. Subdivide $I$ into $k$ equal parts. By Theorems 2.2 and 2.3 and Lemma 6.5, $B_{x_{1}}(I)$ and $B_{x_{2}}(I)$ are compact connected nonempty sets.

Further $B_{x_{1}}(a)=B_{x_{2}}(a)$. Thus $B_{x_{1}}(I) \cap B_{x_{2}}(I) \neq \emptyset$ and $X_{k}=B_{x_{1}}(I) \cup B_{x_{2}}(I)$ is connected. Now $B_{x_{1}}(b)=\left\{x_{1}\right\}$ and $B_{x_{2}}(b)=\left\{x_{2}\right\}$. Thus $x_{1}$ and $x_{2} \in X_{k}$. Let $G_{1}$ and $G_{2}$ be disjoint open sets in $C(I)$ such that $H_{1} \subseteq G_{1}$ and $H_{2} \subseteq G_{2}$. Then there exists a function $y_{k} \in X_{k}$ which is not contained in either $G_{1}$ or $G_{2}$.

Now $y_{k}$ is of the form

(i) $y_{k}(t)=x_{i}(t)$ for all $t \in\left[a, r_{k}\right]$,

(ii) $y_{k}^{\prime}(t) \in F\left(t, y_{k}\left(t_{i}\right)\right)$ almost everywhere $t \in\left[r_{k}, b\right]$ where $t \in\left[t_{i}, t_{i+1}\right)$.

Thus $y_{k}(a)=x_{0}$ and $\left|y_{k}^{\prime}(t)\right| \leq g(t)$. We now let $k$ (the number of subdivisions of $I$ ) vary to obtain a sequence $\left\{y_{k}\right\}$. These $y_{k}$ are bounded and equicontinuous. By Ascoli's Theorem there is a convergent subsequence, also denoted by $\left\{y_{k}\right\}$, such that $y_{k}$ tends to $y$. Therefore $y$ is not contained in $H\left(a, x_{0}\right)$.

But $y(a)=\lim _{k \rightarrow \infty} y_{k}(a)=x_{0}$. Define $z_{k}: I \rightarrow R^{n}$ by 
(i) $z_{k}(t)=y_{k}(t)$ if $t \in\left[a, r_{k}\right]$,

(ii) $z_{k}(t)=y_{k}\left(t_{i}\right)$ if $t \in\left[r_{k}, b\right]$ and $t \in\left[t_{i}, t_{i+1}\right)$.

Thus $y_{k}^{\prime}() \in F\left(t, z_{k}(t)\right)$ almost everywhere $t \in I$ and $z_{k}$ tends to $y$. Therefore

$$
\begin{aligned}
y^{\prime}(t) & \leftarrow \prod_{i=1}^{\infty} \overline{c o} \underset{k=i}{\bigcup} y_{k}^{\prime}(t) \text { almost everywhere } t \in I \\
& \subseteq \bigcap_{i=1}^{\infty} \overline{c o} \underset{k=i}{U} F\left(t, z_{k}(t)\right) \text { almost everywhere } t \in I \\
& \subseteq F(t, y(t)) \text { almost everywhere } t \in I,
\end{aligned}
$$

by Theorems 4.1 and 2.8. Thus $y \in H\left(a, x_{0}\right)$ which gives us a

contradiction. Therefore $H\left(a, x_{0}\right)$ is connected. //

COROLLARY 6.7. Let $M$ be a connected subset of $I \times R^{n}$. Then $H(M)$ is a connected subset of $C(I)$.

Proof. Theorems $6.6,5.3$ and 2.3 give the required result.

\section{Peripheral attainability and the bang-bang property}

If a point on the boundary of the funnel can be reached by a solution lying on the boundary of the funnel, the point is said to be peripherally attainable. In this section we prove that every point on the boundary of the funnel is peripherally attainable.

$$
\text { Let } \begin{aligned}
t_{0} & \in I \text { and } x_{0} \in R^{n} \text {. Define } \\
& \quad 2\left(t_{0}, x_{0}\right)=\left\{(t, x(t)): t \in I, x \in H\left(t_{0}, x_{0}\right)\right\} .
\end{aligned}
$$

Let $t \in I$. Define

$$
A\left(t, t_{0}, x_{0}\right)=\left\{x(t): x \in H\left(t_{0}, x_{0}\right)\right\} .
$$

$2\left(t_{0}, x_{0}\right)$ is called the solution funnel and $A\left(t, t_{0}, x_{0}\right)$ is called the solution cross-section.

The next two theorems follow easily from these definitions and the preceding theorems. 
THEOREM 7.1. $Z$ maps from $I \times R^{n}$ to $\Omega\left(I \times R^{n}\right)$ and is upper semicontinuous. If $M$ is a connected subset of $I \times R^{n}$ then $Z(M)$ is connected.

THEOREM 7.2. A maps from $I \times I \times R^{n}$ to $\Omega\left(R^{n}\right)$. For $a Z z$ $t \in I$, the map $\left(t_{0}, x_{0}\right) \leftrightarrow A\left(t, t_{0}, x_{0}\right)$ is upper semicontinuous. For all $\left(t_{0}, x_{0}\right) \in I \times R^{n}$ the map $t \rightarrow A\left(t, t_{0}, x_{0}\right)$ is continuous. If $M$ is a connected subset of $I \times R^{n}$, then $A(t, M)$ is connected.

We now use Corollary 6.7 to show that every point on the boundary of the funnel is peripherally attainable.

THEOREM 7.3. Let $K \in \Omega\left(R^{n}\right)$. If $a \in \partial A(b, a, k)$, then there exists $x \in H(a, K)$ such that $x(b)=q$ and $x(t) \in \partial A(t, a, K)$ for $a$ ll $t \in I$.

Proof. (a) We first prove that there exists $x \in H(a, K)$ such that $x(b)=q$ and $x(a) \in \partial K$. Suppose that int $K$ is nonempty (otherwise there is nothing to prove). Let $y_{k}$ be an exterior point of $A(b, a, K)$ such that $\left|y_{k}-q\right|<1 / k$. Let $\overline{y_{k} q}$ denote the closed line segment joining $y_{k}$ and $q \cdot \overline{y_{k} q}$ is connected. Therefore $k_{1}=A\left(a, b, \overline{y_{k} q}\right)$ is connected. Now $k_{1} \cap K^{C} \neq \emptyset$ since $y_{k} \mid A(b, a, K)$. Also $k_{1} \cap K \neq \emptyset$ since $q \in A(b, a, K)$. If $K_{1} \cap \partial K=\varnothing$, then $K_{1} \cap$ int $K \neq \emptyset$ and $K_{1} \subseteq K^{c} \cap$ int $K$. But $K^{c}$ and int $K$ are disjoint nonempty open sets. This is a contradiction since $K_{1}$ is connected. Therefore $K_{1} \cap \partial K \neq \emptyset$. Thus there exists $x_{k} \in H(a, K)$ such that $x_{k}(a) \in \partial K$ and $x_{k}(b) \in \overline{y_{k} q}$. Hence $\left|x_{k}(b)-q\right|<1 / k$. By Theorem 5.1 there exists a convergent subsequence, also denoted by $\left\{x_{k}\right\}$, converging to $x \in H(a, K)$. Therefore $x(b)=q$. Also $x(a) \in \partial K$ since $\partial K$ is closed.

(b) Subdivide $I=[a, b]$ into $k$ equal parts by $t_{i}=a+i \frac{b-a}{k}$. 
Using part (a) we can find $\left.x_{k} \in H(a, k)\right|_{\left[t_{k-1}, b\right]}$ such that $x_{k}(b)=q$, $x_{k}\left(t_{k-1}\right) \in \partial A\left(t_{k-1}, a, k\right)$. Again using (a) we can extend to $\left.x_{k} \in H(a, K)\right|_{\left[t_{k-2}, b\right]}$ such that $x_{k}\left(t_{k-2}\right) \in \partial A\left(t_{k-2}, a, k\right)$. Continuing in this manner we obtain $x_{k} \in H(a, K)$ such that $x_{k}\left(t_{i}\right) \in \partial A\left(t_{i}, a, k\right)$, $i=0, \ldots, k-1$ and $x_{k}(b)=q$. By Theorem 5.1 there exists $a$ subsequence, also denoted by $\left\{x_{k}\right\}$, converging to $x \in H(a, K)$. Therefore $x(t) \in \partial A(t, a, K)$ for all $t \in I$ and $x(b)=q . \quad / /$

If $x(t) \in \partial A(t, M)$ for all $t \in I$ implies that $x^{\prime}(t) \epsilon \partial F(t, x(t))$ almost everywhere $t \in I$, the generalized differential equation $x^{\prime}(t) \in F(t, x(t))$ is said to have the bang-bang property. In [9] Kikuchi proves that if $F$ is continuous in $x$ then $F$ has the bang-bang property.

The assumption of continuity is needed, as is shown by the following example. Let $I=[0,1]$. Define

$$
F(t, x)= \begin{cases}{[0,2]} & \text { if } x=t, \\ \{0\} & \text { otherwise. }\end{cases}
$$

Consider

$$
\begin{aligned}
x^{\prime}(t) & \in F(t, x(t)) \text { almost everywhere } t \in[0,1] . \\
x(0) & =0 .
\end{aligned}
$$

The peripheral solutions are $x_{1}(t)=t$ and $x_{2}(t)=0$. Thus $x_{1}^{\prime}(t)=1$ is not on the boundary of $[0,2]$.

\section{References}

[1] Robert J. Aumann, "Integrals of set-valued functions", J. Math. AnaZ. App Z. 12 (1965), 1-12.

[2] Claude Berge, Espaces topologiques: fonations multivoques (Dunod, Paris, 1959). 
[3] Charles Castaing, "Sur les équations différentielles multivoques", C.R. Acad. Sci. Paris, Sér. A-B 263 (1966), A63-A66.

[4] W.A. Coppel, Stability and asymptotic behaviour of differential equations (D.C. Heath, Boston, 1965).

[5] Nelson Dunford and Jacob T. Schwartz, Linear operators, Part I (Interscience [John Wiley \& Sons], New York, London, 1958).

[6] A.F. Filippov, "Differential equations with many-valued discontinuous right-hand side", Soviet Math. Dokz. 4 (1963), 941-945.

[7] Norio Kikuchi, "Control problems of contingent equation", Publ. Res. Inst. Math. Sci., Kyoto Univ., Ser. A 3 (1967), 85-99.

[8] Norio Kikuchi, "On some fundamental theorems of contingent equations in connection with the control problems", Publ. Res. Inst. Math. Sci., Kyoto Univ., Ser. A 3 (1967), 177-201.

[9] Norio Kikuchi, "On contingent equations satisfying the Carathéodory type conditions", Publ. Res. Inot. Math. Sci., Kyoto Univ., Ser. A 3 (1968), 361-371.

[10] A. Lasota, Z. Opial, "An application of the Kakutani-Ky Fan theorem in the theory of ordinary differential equations", Buzz. Acad. Polon. Sci., Sér. Sci. math. astron. phys. 13 (1965), 781-786.

[11] A. PIIs, "Measurable orientor fields", Bull. Acad. Polon. Sci., Sér. Sci. math. astron. phys. 13 (1965), 565-569.

[12] T. Wazewski, "On an optimal control problem", Differential equations and their applications (Proc. Conf., Prague, 1962, 229-242). (Publ. House Czechoslovak Acad. Sci., Prague; Academic Press, New York, London, 1963.)

Department of Mathematics, Institute of Advanced Studies, Australian National University, Canberra, ACT. 